AIP Appilied Physics

\title{
Highly efficient and tunable fluorescence of a nanofluorophore in silica/metal dual shells with plasmonic resonance
}

Xue-Wen Chen, Sailing He, and Wallace C. H. Choy

Citation: J. Appl. Phys. 103, 024301 (2008); doi: 10.1063/1.2830986

View online: http://dx.doi.org/10.1063/1.2830986

View Table of Contents: http://jap.aip.org/resource/1/JAPIAU/v103/i2

Published by the American Institute of Physics.

\section{Related Articles}

Formation of one-dimensional self-assembled silicon nanoribbons on $\mathrm{Au}(110)-(2 \times 1)$

Appl. Phys. Lett. 102, 083107 (2013)

Manipulated electromagnetic losses by integrating chemically heterogeneous components in Fe-based core/shell architecture

J. Appl. Phys. 113, 084312 (2013)

Enhanced thermoelectric performance in graphitic $\mathrm{ZnO}$ (0001) nanofilms

J. Appl. Phys. 113, 083705 (2013)

Exchange resonance in MDM nanolayer systems: Experiment and theory

J. Chem. Phys. 138, 074705 (2013)

Electrochemical hydrogen property improved in nano-structured perovskite oxide LaFeO3 for $\mathrm{Ni} / \mathrm{MH}$ battery J. Appl. Phys. 113, 053305 (2013)

\section{Additional information on J. Appl. Phys.}

Journal Homepage: http://jap.aip.org/

Journal Information: http://jap.aip.org/about/about_the_journal

Top downloads: http://jap.aip.org/features/most_downloaded

Information for Authors: http://jap.aip.org/authors

\section{ADVERTISEMENT}

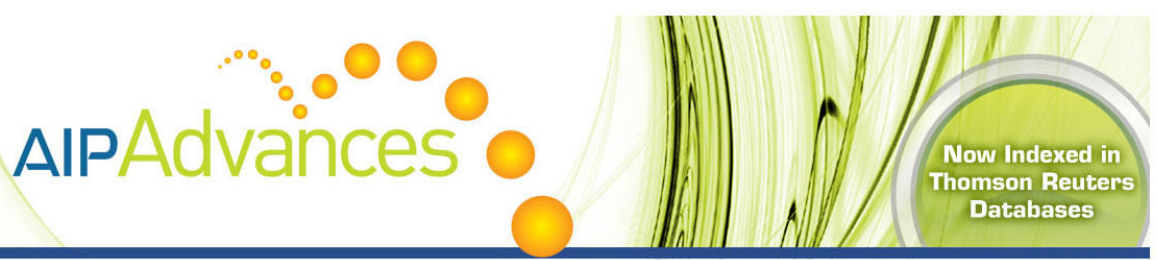

\section{Explore AIP's open access journal: •Rapid publication \\ - Article-level metrics \\ Post-publication rating and commenting}




\title{
Highly efficient and tunable fluorescence of a nanofluorophore in silica/ metal dual shells with plasmonic resonance
}

\author{
Xue-Wen Chen, ${ }^{1}$ Sailing He, ${ }^{1, a)}$ and Wallace C. H. Choy ${ }^{2, b)}$ \\ ${ }^{1}$ Center for Optical and Electromagnetic Research, Zhejiang University; Joint Research Center of Photonics \\ of the Royal Institute of Technology (Sweden) and Zhejiang University, Zijingang campus, Hangzhou \\ 310058, China \\ ${ }^{2}$ Department of Electrical and Electronic Engineering, University of Hong Kong, Pokfulam Road, \\ Hong Kong
}

(Received 24 July 2007; accepted 11 November 2007; published online 16 January 2008)

\begin{abstract}
A dual-shell structure consisting of a silica buffer shell and a metallic outer shell is studied and designed for highly efficient and tunable fluorescence. The enhanced Purcell effect due to the plasmonic resonance and the dependence of the resonance on the structural parameters are analyzed and discussed through a classical electromagnetic approach. With the present dual-shell structure a fivefold enhancement of the fluorescence efficiency can be achieved for a nanofluorophore comprised of multiple tetramethylrhodamine isothiocyanate dye molecules, as compared to the nanofluorophore within a single silica shell. Furthermore, the peak wavelength of the fluorescence spectrum can be tuned easily (due to the variation of the surface plasmon resonance) by over $50 \mathrm{~nm}$ when the refractive index of the ambient material varies from 1.3 to 1.6. (C) 2008 American Institute of Physics. [DOI: 10.1063/1.2830986]
\end{abstract}

\section{INTRODUCTION}

A nanofluorophore encapsulated with a silica shell has recently attracted great interest ${ }^{1-6}$ due to its excellent photochemical stability and biocompatibility in biological and biomedical applications. The nanofluorophore with a diameter of a few nanometers can be a II-VI or III-V compound semiconductor quantum dot, fluorescent proteins, or dye molecules. ${ }^{5,6}$ For fluorescence-based applications, the fluorescence efficiency, i.e., the external quantum efficiency of the nanofluorophore, is an important issue. For the nanofluorophore within a silica shell, the silica shell can protect the nanofluorophore from the solvent and consequently decrease the nonradiative transition rate, and this improves the fluorescence efficiency (see e.g., Ref. 4). However, the fluorescence efficiency still may not be high enough for some applications since the radiative transition rate of the nanofluorophore within a silica shell is much lower than the value in the infinite medium. An alternative and effective way to boost the fluorescence efficiency further is to utilize the Purcell enhancement of the quantum efficiency by increasing the radiative transition rate. ${ }^{7-9}$ It has been reported ${ }^{10}$ that the radiative transition rate can be greatly enhanced through encapsulating the nanofluorophore directly with a metallic shell. However, since the metallic shell introduces large absorption loss when the fluorescent emitter is close to the metallic shell, the fluorescence efficiency for this case is usually still low, particularly for the nanofluorophores studied here with diameters less than $10 \mathrm{~nm}$.

In this paper we study the effect of a dielectric buffer layer between the nanofluorophore and the metallic shell on the radiative transition rate and the fluorescence efficiency,

\footnotetext{
${ }^{a}$ Electronic mail: sailing@zju.edu.cn.

${ }^{b)}$ Electronic mail: chchoy@eee.hku.hk.
}

and show theoretically that such a dual-shell structure as shown in Fig. 1 can give a highly efficient and tunable fluorescence. A variety of chemical and physical methods has been developed for the synthesis of various core-shell nanoparticles with a size-controllable dielectric core and metal shell. $^{4,11,12}$ Thus, the present dual-shell structure with designed parameters should be feasible and practical for synthesis. Although a theoretical study on a two-level atom radiating anywhere inside a spherically layered structure has been reported, ${ }^{13}$ the study was limited to a few special structures with a fixed emission wavelength. Thus, the enhanced Purcell effect, due to the plasmonic resonance and the other related phenomena which will be discussed in this paper, could not be observed in Ref. 13. In addition, unlike the case of a single silica shell or single metallic shell, we expect that a properly designed dual-shell structure can offer simultaneously a large Purcell enhancement of the internal quantum efficiency and a high outcoupling efficiency (i.e., the ratio of the far-field radiation power to the total power emitted from the nanofluorophore), which results in a significant improvement of the fluorescence efficiency. The large Purcell enhancement in the dual-shell structure is due to the resonant excitation of the surface plasmon in the metallic shell, where the resonant wavelength depends on the refractive index $n$ of the ambient material. Thus, we expect the fluorescence spec-

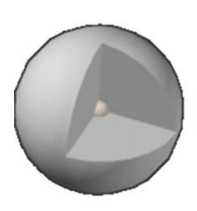

(a)

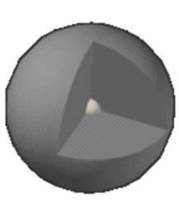

(b)

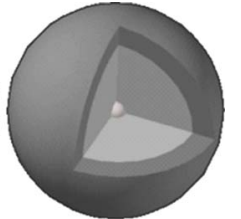

(c)
FIG. 1. Schematic diagrams of a nanofluorophore within (a) a single silica shell; (b) a single metallic shell; and (c) silica/metal dual shells. 
trum to be sensitive to $n$. Here, as examples of practical interest, we study fluorescent emission from a $4 \mathrm{~nm}$ diameter nanofluorophore comprised of multiple tetramethylrhodamine isothiocyanate (TRITC) dye molecules.

The paper is organized as follows. In Sec. II, a theoretical analysis is given on the fluorescence of emitters in a spherically multilayered structure. In Sec. III, we study the dependence of the plasmonic resonance on the structural parameters of the dual-shell structure, optimize the dual-shell structure for highly efficient fluorescence, and show that the fluorescent spectrum can be tuned easily by changing the refractive index of the ambient material. Conclusion will be addressed in Sec. IV.

\section{THOERETICAL ANALYSIS}

The theoretical treatment of the fluorescence is based on the classical electromagnetics with the fluorescent emitter modeled as incoherent classical electric dipoles ${ }^{8,14,15}$ (with a constant current but random orientation in space). The applicability of the classical electromagnetic approach for calculating the radiative transition rate relies on the fact that the local photonic density of states describes the averaged total power emitted from the incoherent classical electric dipoles. ${ }^{14,15}$ Note that in the case of an ultrathin metallic shell, the standard Mie theory may fail since the nonlocal effect associated with the longitudinal surface plasmon modes ${ }^{16-18}$ may occur. This nonlocal effect is considered in our treatment. In order to study the fluorescence properties, one needs to calculate the averaged total emission power $F$ and the far-field radiation power $U$ (both are normalized by the total emission power of an electric dipole in the infinite medium) of the dipoles in the spherically multilayered structure. As a consequence of Fermi's golden rule, the radiative transition rate is modified as ${ }^{7-9}$

$$
\Gamma_{r}^{s}=F \Gamma_{r}^{0},
$$

where $\Gamma_{r}^{0}$ and $\Gamma_{r}^{s}$ are the radiative transition rate in the infinite medium and the spherically multilayered media, respectively. Here, the total emission power $F$ is also called the Purcell factor. Assuming that the nonradiative transition rate in the spherically multilayered media is $\Gamma_{n r}$, the internal quantum efficiency $\eta_{q}^{s}$ and the fluorescence efficiency $\eta_{f}$ are obtained as

$$
\begin{aligned}
& \eta_{q}^{s} \equiv \frac{\Gamma_{r}^{s}}{\Gamma_{r}^{s}+\Gamma_{n r}}=\frac{F}{F \eta_{q}^{0}+\left(1-\eta_{q}^{0}\right)} \eta_{q}^{0}, \\
& \eta_{f} \equiv \eta_{q}^{s} \frac{U}{F}=\frac{U}{F \eta_{q}^{0}+\left(1-\eta_{q}^{0}\right)} \eta_{q}^{0},
\end{aligned}
$$

where $\eta_{q}^{0}=\Gamma_{r}^{0} /\left(\Gamma_{r}^{0}+\Gamma_{n r}\right)$ is the initial internal quantum efficiency. In a fixed short time period, the total number of emitted photons at a given wavelength $\lambda$ is proportional to the radiative transition rate. Thus, the observable fluorescence spectrum $S(\lambda)$ is proportional to the product of the intrinsic fluorescence spectrum $S_{0}(\lambda)$, radiative transition rate, and the outcoupling efficiency $U / F$, i.e. [cf. Eq. (1)],

$$
S(\lambda) \propto S_{0}(\lambda) \times\left[\Gamma_{r}^{s}(\lambda) / \Gamma_{r}^{0}\right] \times[U(\lambda) / F(\lambda)]=S_{0}(\lambda) U(\lambda) .
$$

The fluorescence efficiency averaged over the emission spectrum is given by

$$
\eta=\int \eta_{f}(\lambda) S(\lambda) d \lambda / \int S(\lambda) d \lambda .
$$

For the $4 \mathrm{~nm}$ diameter TRITC nanofluorophore studied here, the intrinsic fluorescent spectrum $S_{0}(\lambda)$ is considered to have a Gaussian shape centered at $575 \mathrm{~nm}$ and a FWHM (full width at half-maximum) of $60 \mathrm{~nm}$ according to the experimental results. ${ }^{4,6}$ In addition, an initial internal quantum efficiency as low as $\eta_{q}^{0}=0.25$ is used for TRITC. ${ }^{19}$ For an electric dipole with a dipole moment $\vec{p}$ in the spherically layered structure, the total emission power $F$ is given by 8,20

$$
F=\left\{\vec{p} \times \operatorname{Im}\left[E_{s}\left(\vec{r}^{\prime}, \vec{r}^{\prime}\right)\right]\right\} /\left\{\vec{p} \times \operatorname{Im}\left[E_{0}\left(\vec{r}^{\prime}, \vec{r}^{\prime}\right)\right]\right\},
$$

where $E_{s}\left(\vec{r}, \vec{r}^{\prime}\right)$ and $E_{0}\left(\vec{r}, \vec{r}^{\prime}\right)$ are the electric field at $\vec{r}$ due to the radiating dipole located at $\vec{r}^{\prime}$ in the spherically layered structure and the infinite medium, respectively. Here, $\operatorname{Im}(\cdot)$ stands for the imaginary part of (.). In the infinite medium, it is well known that

$$
E_{0}\left(\vec{r}, \vec{r}^{\prime}\right)=\left(\stackrel{\leftrightarrow}{I}+\frac{\nabla \nabla}{k^{2}}\right) \vec{p} \frac{e^{i k\left|\vec{r}-\vec{r}^{\prime}\right|}}{4 \pi\left|\vec{r}-\vec{r}^{\prime}\right|},
$$

where $\stackrel{\leftrightarrow}{I}$ is the unitary dyadic and $k$ is the wavenumber in the emitting medium. In order to calculate $E_{s}\left(\vec{r}, \vec{r}^{\prime}\right)$, we decompose $E_{0}\left(\vec{r}, \vec{r}^{\prime}\right)$ in terms of vector spherical harmonics and calculate the reflection and transmission coefficients of each spherical harmonic at the interfaces by matching the boundary conditions. Then, $E_{s}\left(\vec{r}, \vec{r}^{\prime}\right)$ can be evaluated through a summation of the vector spherical harmonics with the consideration of the reflection and transmission coefficients. ${ }^{20}$ According to Eq. (6), the Purcell factor $F$ can be obtained. The far-field radiation power $U$ is an integral of the outgoing time-averaged Poynting power over the outmost spherical surface, which can be readily evaluated through the knowledge of the electric field in the outmost region of the spherically layered structure. ${ }^{20}$

\section{RESULTS AND DISCUSSION}

In this section, we study the dependence of the plasmonic resonance on the structural parameters of the dualshell structure, optimize the dual-shell structure for highly efficient fluorescence, and show that the fluorescence spectrum can be tuned easily by changing the refractive index of the ambient material. In our calculations, the refractive indices of the nanofluorophore core and silica shell are assumed to be 2.0 and 1.46 , respectively, over the fluorescence spectrum range. Two kinds of metal shells, i.e., silver and gold shells, are considered here. For the special configuration of a dielectric core/metallic shell system, a recent report ${ }^{21}$ has shown that the experiment agrees well with the theory without considering the size dependence of the dielectric function of the metal. Thus, according to Refs. 21 and 22 and the references therein, we use the dielectric function of bulk 

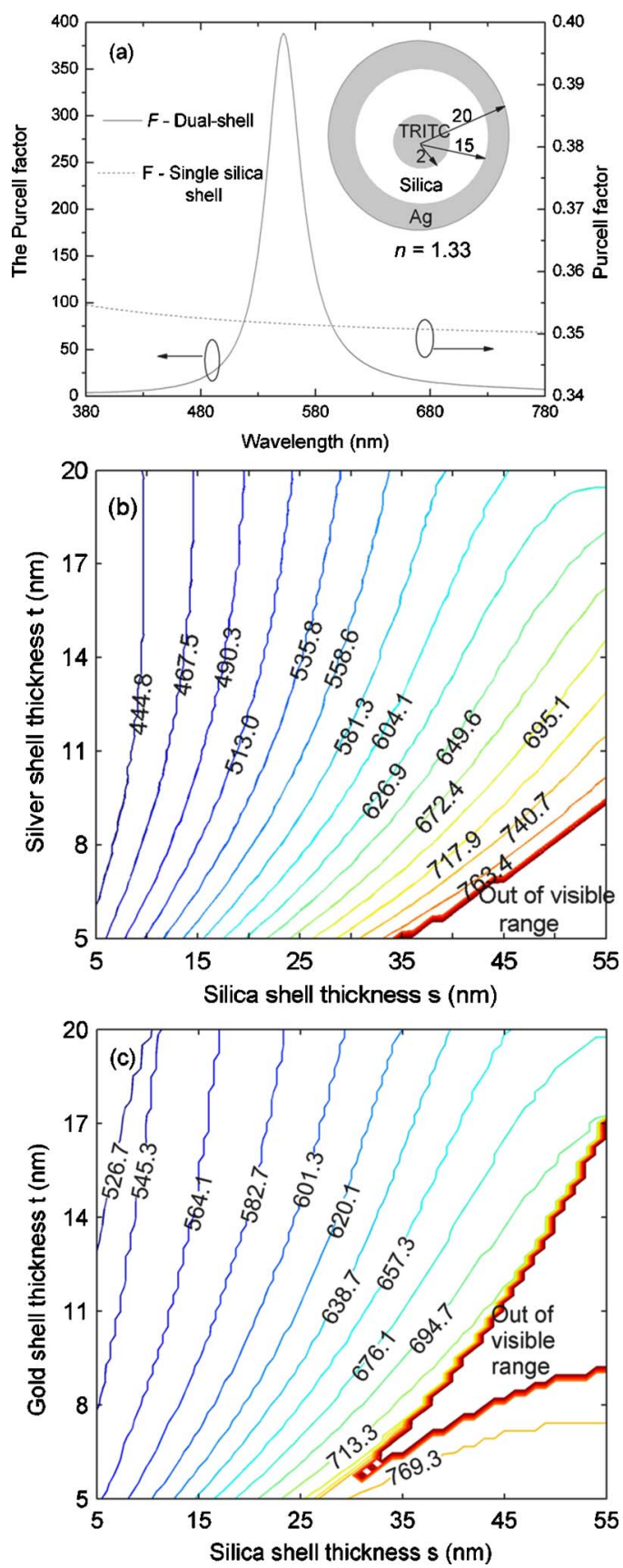

FIG. 2. (Color online) (a) Spectra of the Purcell factor $F$ of the silica/silver dual-shell nanoparticle shown in the figure. (b) Contour plot of the plasmonic resonant wavelength $(\mathrm{nm})$ as a function of the thicknesses of the silica shell and silver shell. (c) Contour plot of the plasmonic resonant wavelength $(\mathrm{nm})$ as a function of the thicknesses of the silica shell and gold shell.

metals $^{23}$ in the calculation. The ambient material of the nanoparticle is assumed to be water with a refractive index of 1.33. Later we will show that the fluorescence spectrum and efficiency depend strongly on the refractive index $n$ of the ambient material.

\section{A. Fluorescence with plasmonic resonance in the dual-shell structure}

In the present dual-shell structure, the radiative transition rate can be greatly enhanced due to the resonant excitation of the surface plasmon in the metallic shell. Figure 2(a) shows the spectrum of the Purcell factor $F$ as the solid line for the dual-shell structure displayed in the figure. For comparison, the spectrum of $F$ for the same structure but without the silver shell is shown as the dashed line in Fig. 2(a). One sees that, different from the nanofluorophore, within a single silica shell the spectrum of $F$ for the dual-shell structure shows a resonant structure and $F$ is enhanced by more than three orders of magnitudes at the resonant wavelength. Thus, the radiative transition rate of the emitters in the dual-shell structure is much faster and consequently the internal quantum efficiency is greatly enhanced as the radiative transition rate outweighs the nonradiative transition rate [see Eq. (2)]. The plasmonic resonance in the dual-shell structure depends strongly on the structural parameters, i.e., the silica shell thickness and the metallic shell thickness. Figure 2(b) shows a contour plot of the plasmonic resonant wavelength as a function of the thickness of the silica shell $s$ and the thickness of the silver shell $t$, where the values on the lines indicate the resonant wavelength (nm). From Fig. 2(b), one observes that for a fixed resonant wavelength a nearly linear relation of $s$ and $t$ should be satisfied. In addition, the resonant wavelength becomes highly sensitive to the silica shell thickness when the silver shell is thin. In the silica/silver dual-shell structure, one can observe that the resonant wavelength has a lower limit of about $440 \mathrm{~nm}$. We also studied the case of the silica/gold dual-shell structure and have shown the results in Fig. 2(c), where similar phenomena are observed. In the silica/gold dual-shell structure, the lower limit of the resonant wavelength is around $525 \mathrm{~nm}$, much longer than that in the silica/silver structure.

\section{B. Optimal design of the dual-shell structure for highly efficient fluorescence}

In this subsection, we optimize the dual-shell structure to achieve a high-efficiency fluorescence. According to Eqs. (3) and (5), we calculate the dependence of the fluorescence efficiency of TRITC on the two structural parameters, i.e., the silica shell thickness $s$ and the metallic shell thickness $t$. Figure 3(a) shows a contour plot of the fluorescence efficiency as a function of $s$ and $t$, where the values on the lines indicate the efficiency. From this figure, one can find the optimal structural parameters $(s, t)$ which correspond to the maximal fluorescence efficiency. At the optimal point $(s, t)$, the large Purcell enhancement of the internal quantum efficiency and high outcoupling efficiency are simultaneously achieved. Figure 3(b) shows the spectra of the fluorescence, the normalized total emission power $F$, and the far-field radiation power $U$ of the optimized silica/silver dual-shell structure. As shown by the dashed line in Fig. 3(b), a large Purcell enhancement of the radiative transition rate due to the resonant excitation of the surface plasmon is observed. Moreover, the peak wavelength of the fluorescence spectrum in Fig. 3(b) does not exactly coincide with the peak of the spectra of $F$ and $U$. This is due to the contribution of the outcoupling efficiency [see Eq. (3)]. Besides the silica/silver dual-shell structures, we also optimized the silica/gold dualshell structures for high-efficiency fluorescence. For comparison, we calculated the fluorescence efficiencies for the 

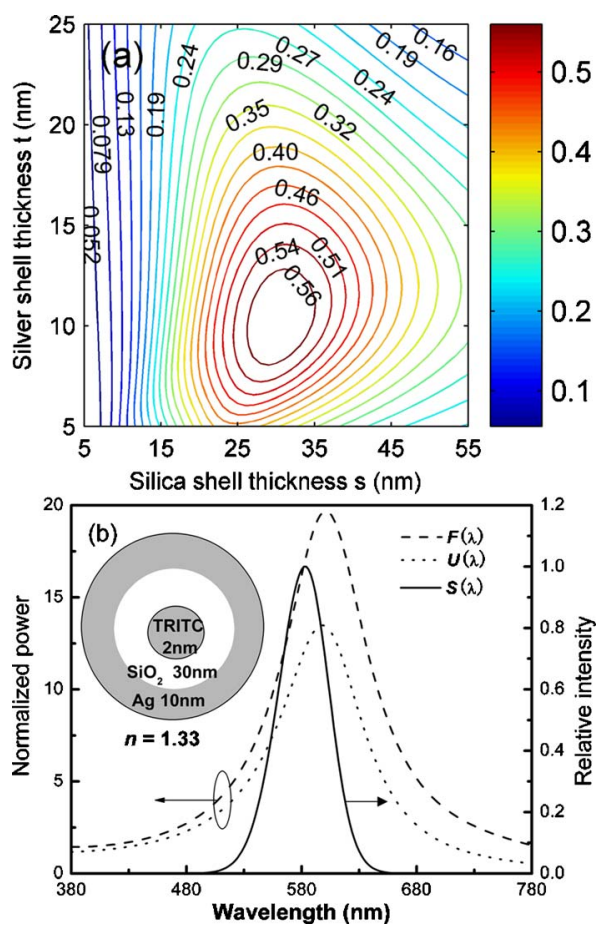

FIG. 3. (Color online) (a) Contour plot of the fluorescence efficiency as a function of the thicknesses of the silica shell and silver shell. (b) Fluorescence spectrum $S(\lambda)$ (solid line), the total emission power $F$ (dotted line), and far-field radiation power $U$ (dashed line) for the optimized dual-shelled nanoparticle shown in the inset of the figure.

nanofluorophore within a single silica shell and the nanofluorophore within an optimized single silver shell. The optimized parameters, the corresponding Purcell factors $F$ at $\lambda$ $=575 \mathrm{~nm}$, and the efficiencies of the four nanoparticles are listed in Table I. One sees that, for the single-silica-shell structure, although the outcoupling efficiency is 1 (i.e., $F$ $=U$ ), the fluorescence efficiency is as low as $10 \%$ since the radiative transition rate is very low. For the optimized singlemetal-shell structure, although the radiative transition rate is over 1052 times larger than the value in the infinite medium, the fluorescence efficiency is less than $1 \%$ because over $99 \%$ of the emitted power is absorbed in the metal shell since the diameter of the nanofluorophore is very small; consequently the fluorescent emitters are very close to the metal shell (the thickness of the metal shell has already been optimized for the maximal fluorescence efficiency). Our dual-shell structure gives simultaneously a large Purcell enhancement of the internal quantum yield and a high outcoupling efficiency, which result in a significant improvement of the fluorescence

TABLE I. Structural parameters, the Purcell factor $F$, and fluorescence efficiency $\eta_{\text {ext }}$ of TRITC within the optimized silica/silver dual-shell structure, the optimized silica/gold dual-shell structure, a single silica shell, and the optimized single silver shell.

\begin{tabular}{ccccc}
\hline \hline & $\begin{array}{c}\text { Silica/silver } \\
\text { dual shells }\end{array}$ & $\begin{array}{c}\text { Silica/gold } \\
\text { dual shells }\end{array}$ & $\begin{array}{c}\text { Single } \\
\text { silica shell }\end{array}$ & $\begin{array}{c}\text { Only } \\
\text { silver shell }\end{array}$ \\
\hline Silica shell & $30 \mathrm{~nm}$ & $30 \mathrm{~nm}$ & $13 \mathrm{~nm}$ & 0 \\
Metal shell & $10 \mathrm{~nm}$ & $14 \mathrm{~nm}$ & 0 & $5 \mathrm{~nm}$ \\
$F(\lambda=575 \mathrm{~nm})$ & 14.5 & 5.97 & 0.35 & 1053 \\
$\eta_{\text {ext }}$ & 0.588 & 0.403 & 0.10 & $<0.01$ \\
\hline \hline
\end{tabular}
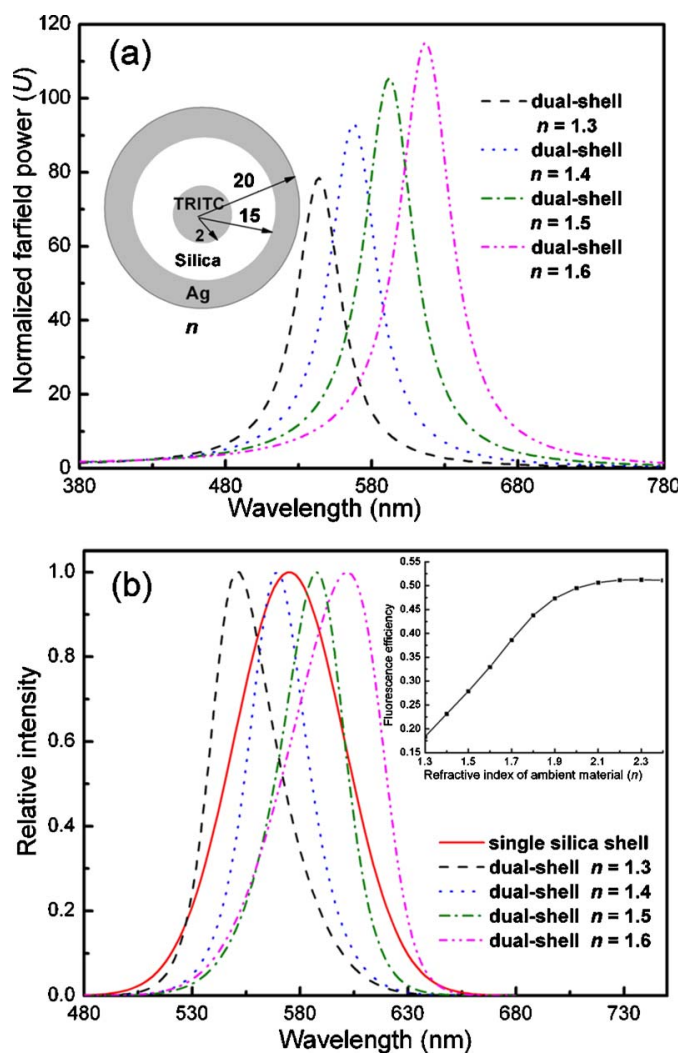

FIG. 4. (Color online) (a) Spectra of the far-field radiation power $U$. (b) Fluorescence spectra of the dual-shell nanoparticle shown in the inset of (a) for different refractive indices $(n)$ of the ambient material. Inset of $(b)$ shows the dependence of the fluorescence efficiencies on the refractive index of the ambient material.

efficiency. One sees that for the optimized silica/silver dualshell structure, the fluorescence efficiency is $58.8 \%$, which is roughly five times larger than the efficiency for the singlesilica-shell structure. The fluorescence efficiency for the optimized silica/gold dual-shell structure is about $40.3 \%$, which is less efficient than that of the silica/silver dual-shell structure. Thus, from the point of view of efficiency, silver is better for use as the metallic outer shell in our dual-shell structure to achieve high-efficiency fluorescence for TRITC.

\section{Tunable fluorescence}

In this subsection, we will show that the fluorescent spectrum of the nanofluorophore in the dual-shell structure is sensitive to the refractive index $n$ of the ambient material. According to Eq. (4), the observable fluorescence spectrum $S(\lambda)$ is proportional to the product of $U(\lambda)$ and $S_{0}(\lambda)$. Thus, the fluorescence spectrum will change if $U(\lambda)$ significantly changes with $n$. Here, $U(\lambda)$ indeed is sensitive to the refractive index of the ambient material since the plasmonic resonant wavelength depends on $n$. For the dual-shell structure displayed in Fig. 4(a), the spectra of $U(\lambda)$ for various $n$ are calculated and plotted in the figure. As $n$ increases, the peak wavelength of $U(\lambda)$ shifts to a longer wavelength. Since the fluorescence spectrum is directly related to $U(\lambda)$, the fluorescence spectrum should show a redshift as $n$ increases. We plot the fluorescence spectra in Fig. 4(b) with various line styles for various $n$. As $n$ varies from 1.3 to 1.6 , the peak 
wavelength of the fluorescence spectrum is redshifted by over $50 \mathrm{~nm}$. For comparison, the fluorescence spectrum for the $4 \mathrm{~nm}$ diameter TRITC nanofluorophore within a single silica shell is shown in Fig. 4(b) by the solid line, which looks much broader and has no spectral shift when the refractive index $n$ varies. Meanwhile, the fluorescence efficiency, as shown in the inset of Fig. 4(b), also significantly changes as $n$ increases. The sensitivity of the fluorescence spectrum and efficiency to the ambient material offers extra flexibilities and may broaden its applications in biological sensing and labeling. For different types of biologic tissues (as the ambient material), the refractive indices may vary and consequently the fluorescence spectra (as well as the efficiency) of our dual-shell structure will be distinct. The tuning range can be extended further if the dual-shell structure is optimized.

\section{CONCLUSIONS}

In conclusion, a nanofluorophore core with a buffer shell (silica shell) and a metallic outer shell has been studied and designed for highly efficient and tunable fluorescence. The enhanced Purcell effect due to plasmonic resonance and the variation of the resonance with structural parameters have been analyzed and discussed thoroughly through a classical electromagnetic approach. With our dual-shell structure, a nearly fivefold enhancement of the fluorescence efficiency has been achieved as compared with the nanofluorophore within a single silica shell. Furthermore, unlike the nanofluorophore within a single silica shell, the peak of the fluorescence spectrum can be easily tuned by over $50 \mathrm{~nm}$ when the refractive index of the ambient material varies from 1.3 to 1.6. The significant enhancement and tunability of the fluorescence facilitate and broaden the applications of our dualshell nanoparticles in biological sensing and labeling.

\section{ACKNOWLEDGMENTS}

This work is supported partially by the National Basic Research Program (973) of China (2004CB719800), the National Natural Science Foundation of China (60688401), the Seed Fund (No. 10207444) of the University of Hong Kong and a grant (No. 14300.324.01) from the Research Grant Council of the Hong Kong Special Administrative Region, China.

${ }^{1}$ S. Santra, K. Wang, R. Tapec, and W. Tan, J. Biomed. Opt. 6, 160 (2001).

${ }^{2}$ X. J. Zhao, R. P. Bagwe, and W. H. Tan, Adv. Mater. 16, 173 (2004).

${ }^{3}$ X. C. Zhou and J. Z. Zhou, Anal. Chem. 76, 5302 (2004).

${ }^{4}$ H. Ow, D. R. Larson, M. Srivastava, B. A. Baird, W. W. Webb, and U. Wiesner, Nano Lett. 5, 113 (2005).

${ }^{5}$ F. Wang, W. B. Tan, Y. Zhang, X. P. Fan, and M. Q. Wang, Nanotechnology 17, R1 (2006).

${ }^{6}$ A. Burns, H. Ow, and U. Wiesner, Chem. Soc. Rev. 35, 1028 (2006).

${ }^{7}$ E. M. Purcell, Proceedings of the American Physical Society in Physical Review (1946), Vol. 69, p. 681.

${ }^{8}$ W. Lukosz, Phys. Rev. B 22, 3030 (1980).

${ }^{9}$ K. Okamoto, I. Niki, A. Shvartser, Y. Narukawa, T. Mukai, and A. Scherer, Nat. Mater. 3, 601 (2004).

${ }^{10}$ J. Enderlein, Appl. Phys. Lett. 80, 315 (2002).

${ }^{11}$ S. J. Oldenburg, R. D. Averitt, S. L. Westcott, and N. J. Halas, Chem. Phys. Lett. 288, 243 (1998).

${ }^{12}$ C. Graf and A. van Blaaderen, Langmuir 18, 524 (2002).

${ }^{13}$ A. Moroz, Chem. Phys. 317, 1 (2005).

${ }^{14}$ A. F. Koenderink, M. Kafesaki, C. M. Soukoulis, and V. Sandoghdar, Opt. Lett. 30, 3210 (2005).

${ }^{15}$ R. K. Lee, Y. Xu, and A. Yariv, J. Opt. Soc. Am. B 17, 1438 (2000).

${ }^{16}$ A. R. Melnyk and M. J. Harrison, Phys. Rev. B 2, 835 (1970).

${ }^{17}$ P. T. Leung, Phys. Rev. B 42, 7622 (1990).

${ }^{18}$ A. Pack, M. Hietschold, and R. Wannemacher, Opt. Commun. 194, 277 (2001).

${ }^{19}$ V. K. Yadavalli, R. J. Russell, M. V. Pishko, M. J. McShane, and G. L. Cote, Sens. Actuators B Chem. 105, 365 (2005).

${ }^{20}$ X. W. Chen, W. C. H. Choy, S. L. He, and P. C. Chui, Opt. Express 15, 7084 (2007)

${ }^{21}$ C. L. Nehl, N. Grady, G. P. Goodrich, F. Tam, N. J. Halas, and J. H. Hafner, Nano Lett. 4, 2355 (2004).

${ }^{22}$ J. Enderlein, Appl. Phys. Lett. 87, 066102 (2005).

${ }^{23}$ E. D. Palik, Handbook of Optical Constants of Solids (Academic, Boston, 1985). 